FIAN/TD-19/95

ITEP/TH-4/96

\title{
Exact solutions to quantum field theories and integrable equations $\mathrm{g}$
}

\author{
A.Marshakov \\ Theory Department, P. N. Lebedev Physics Institute, Leninsky prospect, 53, Moscow, 117924, Russia \\ and \\ ITEP, Moscow 117259, Russia
}

\begin{abstract}
The exact solutions to quantum string and gauge field theories are discussed and their formulation in the framework of integrable systems is presented. In particular I consider in detail several examples of appearence of solutions to the first-order integrable equations of hydrodynamical type and stress that all known examples can be treated as partial solutions to the same problem in the theory of integrable systems.
\end{abstract}

\footnotetext{
${ }^{1}$ talk given at: Singular limits of nonlinear dispersive waves, September 4-9 1995, Zvenigorod, Russia and Supersymmetries and quantum symmetries, September 25-30, Dubna, Russia

${ }^{2}$ E-mail address: mars@lpi.ac.ru
} 


\section{Introduction}

In this note I will try to describe some recent advances in modern quantum field theory related with the appearence of several examples of the exact quantum solutions. Amazingly enough it turns out that an adequate language for the description of the exactly solved quantum theories can be found in the framework of classical integrable systems and some essential role is played by rather simple first order equations of hydrodynamical type or so called Whitham equations on moduli spaces of complex structures of Riemann surfaces appearing when solving (quasi)periodic problems for the systems of KP or Toda type.

The original problem in quantum field theory defined by the "bare" action functional

$$
S=\int d^{D} x \mathcal{L}\left(\varphi, \partial_{\mu} \varphi, \ldots ; g_{k}\right)
$$

is to compute the exact quantum correlation function

$$
\left\langle\Phi_{k_{1}} \ldots \Phi_{k_{n}}\right\rangle=\int D \varphi e^{-\frac{1}{\hbar} S} \Phi_{k_{1}}\left(\varphi, \partial_{\mu} \varphi, \ldots\right) \ldots \Phi_{k_{n}}\left(\varphi, \partial_{\mu} \varphi, \ldots\right) \equiv F_{k_{1}, \ldots, k_{n}}(g, u)
$$

as a function of coupling constants $\left\{g_{k}\right\}$, quantum characteristics of physical operators $\left\{k_{1}, \ldots, k_{n}\right\}$ (in general, of course, not being just a discrete set of numbers) and possible parameters of classical solutions $\left\{u_{\alpha}\right\}$ or moduli of the theory.

Unfortunately almost never the integral in (2) can be computed exactly. Up to now the only working methods in quantum field theory are given by perturbation theory and lattice regularization of the problem (2) resulting usually in some approximations being quite far from the exact answer. However, sometimes it turns out that it is possible to propose the exact answer not computing the path integral directly and there exists a beleif that such answers are nevertheless true.

First, this is the case of some models of string theory. Using an elegant discretization which is quite far from an original $D=2$ Lagrangian it has been possible to compute exact solutions to the nonperturbative quantum string correlators in the technique of matrix models. An important point is that the answer was proposed in the form of a solution to the hierarchy of classical integrable equations. The most advanced results were obtained for so called topological $2 D$ field theories where it has turned to be possible to get the solutions explicitely. 1

Second, there are examples of the exact solutions in $4 D$ supersymmetric gauge theories [2, 3] deduced from the investigation of duality properties and an assumption of complex analiticity of quantum answers as functions of coupling constants and/or moduli parameters. An important observation is 四 that the proposed exact answer is again a particular solution to certain differential equation.

Below I will try to pay attention to this universal property of the exact quantum field theory solutions. It will be demonstrated that various exact quantum answers to different theories can be obtained as different solutions almost to the same classical problem of (integrable) differential equations. Unfortunately it is still unclear what is the concrete mechanism of arising these equation in quantum field theory.

The main hope is that the (first - order) equations of hydrodynamical type are generalizations of the renormalization-group technique of the perturbation theory. Indeed it is known that in the perturbation the-

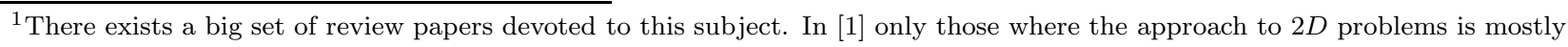
closed to that developed below are presented (see also references therein).
} 
ory the scale dependence of quantum correlation functions is related with their dependence on the coupling constants by means of the first order equation

$$
\left(\frac{d}{d \log \mu}-\sum \beta_{i}(g) \frac{\partial}{\partial g_{i}}\right) F(g ; \mu)=0
$$

Naively in the exact solution there is no scale dependence, so the resulting equation could be of the form $\sum \beta_{i}(g) \frac{\partial F}{\partial g_{i}}=0$, or the derivatives over $\mu$ could be replaced by the derivatives over moduli $\left\{u_{\alpha}\right\}$. Even accepting this it is necessary to stress that there is no known way to define $\beta$-function beyond the perturbation theory (i.e. not using the scale dependence $\beta_{i}(g)=\frac{\partial g_{i}}{\partial \log \mu}$ ). One can also hope to find integrable structures in the nonperturbative calculations investigating the moduli spaces of classical solutions (like instantons in gauge theories or $2 D \sigma$-models) and this way may also lead towards the Hitchin-type constructions [5].

In what follows I will concentrate mostly on two examples of quantum exact solutions. The first one is the simplest case of $D=2$ topological string theory - pure topological gravity where the exact generating function of all quantum correlators is known [6]. In this example the correlators $F_{k_{1} \ldots k_{n}}$ are just integer numbers - so called intersection indices on moduli spaces of complex structures of Riemann surfaces. The second (almost a realistic) example is supersymmetric Yang-Mills theory. It is not yet possible to get all quantum answers in the latter case but what was proposed recently in [2] is the exact nonperturbative dependence of the coupling constants $\tau=\theta+\frac{i}{g^{2}}=\tau(u)$ where (the bare) constants can be read off the Lagrangian

$$
\mathcal{L}=\frac{1}{g^{2}} \operatorname{Tr} F_{\mu \nu}^{2}+i \theta \operatorname{Tr} F_{\mu \nu} \tilde{F}_{\mu \nu}+\ldots
$$

on the moduli of the exact solution $\left\{u_{\alpha}\right\}$ - vacuum expectation values of the Higgs field as well as the allowed massive spectrum of the theory

$$
M=\left|\mathbf{n a}(u)+\mathbf{m a}_{D}(u)\right|=\left|\left(\mathbf{n} \oint_{\mathbf{A}}+\mathbf{m} \oint_{\mathbf{B}}\right) d S\right|
$$

and the effective action of the "light modes" determined by

$$
a_{D, i}=\frac{\partial \mathcal{F}}{\partial a_{i}} \quad \tau_{i j}=\frac{\partial a_{D, i}}{\partial a_{j}}=\frac{\partial^{2} \mathcal{F}}{\partial a_{i} \partial a_{j}}
$$

Now we will see that the above formulas can be considered as different solutions of the same problem concerning classical integrable equations.

There are two main questions concerning the appearence of the integrable equations. First one - how the effective action of a quantum field theory becomes related with the fundamental object in the theory of integrable systems $\mathcal{F}=\log \mathcal{T}$ - the $\tau$-function of an integrable hierarchy and what is the mechanism expressing the data (5) in terms of the generating differential $d S$ of the Whitham hierarchy [4]. The second question arises since the Whitham equations have in principle lots of solutions - so what is the way to distinguish the "physical" ones appearing in the vicinity of a finite-gap solutions to integrable systems of a KP/Toda-type. Therefore, one should learn what kind of spectral curves (i.e. the finite-dimensional integrable systems) arise in the context of quantum field theory and discuss the particular solutions determined by these spectral curves. 


\section{Whitham equations}

Let us briefly remind the most general definition of the Whitham hierarchy given by Krichever [7]. One has a local system of functions $\Omega_{A}$ on one-dimensional complex curve and the corresponding set of parameters $t_{A}$ so that it is possible to introduce a 1 -form in the space with co-ordinates $\{\lambda, t\}$ where $\lambda$ is a local parameter on a curve 2

$$
\omega=\sum \Omega_{A} \delta t_{A}
$$

The Whitham equations are

$$
\delta \omega \wedge \delta \omega=0 \quad \delta \omega=\partial_{\lambda} \Omega_{A} \delta \lambda \wedge \delta t_{A}+\partial_{B} \Omega_{A} \delta t_{A} \wedge \delta t_{B}
$$

so that one needs to check the independent vanishing of the two different terms $-\delta t^{4}$ and $\delta t^{3} \delta \lambda$. The second term gives

$$
\sum \partial_{\lambda} \Omega_{A} \partial_{B} \Omega_{C}=0
$$

for the antisymmetrized sum or introducing some explicit co-ordinates $t_{A_{0}} \equiv x$ and $\Omega_{A_{0}}(z, t) \equiv p(z, t)$ is a local co-ordinate adjusted to the fixed choice of parameters $\left\{t_{A}\right\}$

$$
\partial_{A} \Omega_{B}-\partial_{B} \Omega_{A}+\left\{\Omega_{A}, \Omega_{B}\right\}=0 \quad\left\{\Omega_{A}, \Omega_{B}\right\} \equiv \frac{\partial \Omega_{A}}{\partial x} \frac{\partial \Omega_{B}}{\partial p}-\frac{\partial \Omega_{B}}{\partial x} \frac{\partial \Omega_{A}}{\partial p}
$$

In fact (10) strongly depend on the choice of the local co-ordinate $p$. The equations (8), (9) and (10) are defined only locally and have huge amount of solutions.

A possible way to restrict ourselves is to get "globalized picture" related with the "modulation" of parameters of the finite-gap solutions of integrable systems of KP-Toda type. The KP $\tau$-function associated with a given spectral Riemann curve is

$$
\mathcal{T}\left\{t_{i}\right\}=e^{\sum t_{i} \gamma_{i j} t_{j}} \vartheta\left(\mathbf{\Phi}_{0}+\sum t_{i} \mathbf{k}_{i}\right) \quad \mathbf{k}_{i}=\oint_{\mathbf{B}} d \Omega_{i}
$$

where $\vartheta$ is a Riemann theta-function and $d \Omega_{i}$ are meromorphic 1-differentials with poles of the order $i+1$ at a marked point $z_{0}$. They are completely specified by the normalization relations

$$
\oint_{\mathbf{A}} d \Omega_{i}=0
$$

and the asymptotic behaiviour

$$
d \Omega_{i}=\left(\xi^{-i-1}+o(\xi)\right) d \xi
$$

where $\xi$ is a local coordinate in the vicinity of $z_{0}$. The moduli $\left\{u_{\alpha}\right\}$ of spectral curve are invariants of KP flows,

$$
\frac{\partial u_{\alpha}}{\partial t_{i}}=0
$$

The way the moduli depend on $t_{i}$ after the "modulation" is defined by the Whitham equations (10). For $z=z(\lambda, t)$ so that $\partial_{i} z=\left\{\Omega_{i}, z\right\}$ they acquire more simple form

$$
\frac{\partial d \Omega_{i}(z)}{\partial t_{j}}=\frac{\partial d \Omega_{j}(z)}{\partial t_{i}}
$$

\footnotetext{
${ }^{2}$ Actually it might be better to write

$$
\tilde{\omega}=\omega+\Omega_{\lambda} d \lambda
$$
}


and imply that

$$
d \Omega_{i}(z)=\frac{\partial d S(z)}{\partial t_{i}}
$$

and the equations for moduli, following from (15), are:

$$
\frac{\partial u_{\alpha}}{\partial t_{i}}=v_{i j}^{\alpha \beta}(u) \frac{\partial u_{\beta}}{\partial t_{j}}
$$

with some (in general complicated) functions $v_{i j}^{\alpha \beta}$.

In the KdV (and Toda-chain) case all the spectral curves are hyperelliptic, and for the KdV $i$ takes only odd values $i=2 j+1$, and

$$
d \Omega_{2 j+1}(z)=\frac{\mathcal{P}_{j+g}(z)}{y(z)} d z,
$$

the coefficients of the polynomials $\mathcal{P}_{j}$ being fixed by normalization conditions (12), 13) (one usualy takes $z_{0}=\infty$ and the local parameter in the vicinity of this point is $\xi=z^{-1 / 2}$ ). In this case the equations (17) can be diagonalized if the co-ordinates $\left\{u_{\alpha}\right\}$ on the moduli space are taken to be the ramification points:

$$
v_{i j}^{\alpha \beta}(u)=\left.\delta^{\alpha \beta} \frac{d \Omega_{i}(z)}{d \Omega_{j}(z)}\right|_{z=u_{\alpha}}
$$

Now, an important point is that the differential $d S(z)$ (16) can be constructed for a generic finite-gap solution, i.e. for a solution determined by a (spectral) complex curve [8]. In general, it is a meromorphic differential whose singularities are determined by physical properties of the theory. Below several known examples of exact solutions will be discussed and the corresponding generating differentials (16) will be presented.

\section{Spherical solutions and topological gravity}

Let us, first, turn to the simplest nontrivial solutions of the systems (10), (15) related with $2 D$ topological string theories given at least locally by the formulas

$$
z(\lambda, t)^{p}=\lambda^{p}+u_{p-2}(t) \lambda^{p-2}+\ldots+u_{0}(t)
$$

so that

$$
\Omega_{i}(\lambda, t)=z(\lambda, t)_{+}^{i} \quad \Omega_{i}(z, t)=z^{i}+O\left(z^{\frac{i}{n}-1}\right)
$$

and in the $p=2$ example

$$
\begin{gathered}
z^{2}=\lambda^{2}+U(x, t) \quad \Omega_{i}(\lambda, t)=z(\lambda, t)_{+}^{i}=\left(\lambda^{2}+U(x, t)\right)_{+}^{\frac{1}{2}} \\
\Omega_{i}(z, t)=z^{i}+O\left(z^{\frac{i}{2}-1}\right)
\end{gathered}
$$

Now, in the simplest "global picture" it is possible to interpret $\lambda$ and $z$ as global co-ordinates on sphere with one marked point (where $\lambda=z=\infty$ ).

For particular choice of parameters $\left\{t_{k}\right\}$ this generic picture gives Whitham solutions coming from the $\mathrm{KP} /$ Toda equations in the following way. Starting with the "zero-gap" solution to the KdV hierarchy

$$
\begin{gathered}
U(x, t)=u=\text { const } \quad \Psi(\lambda, t)=e^{\sum_{k>0} t_{k} z^{k}(\lambda)_{+}} \\
\left(\partial_{t_{1}}^{2}+u\right) \Psi=z^{2} \Psi \quad \partial_{t_{k}} \Psi=\left(\partial_{t_{1}}^{2}+u\right)_{+}^{\frac{k}{2}} \Psi
\end{gathered}
$$


one comes by

$$
\begin{gathered}
\Omega_{1}=\overline{\log \Psi(\lambda, t)_{t_{1}}}=z(\lambda)_{+}=\lambda=\sqrt{z^{2}-u} \equiv p(z) \\
\Omega_{i}=\overline{\log \Psi(\lambda, t)_{t_{i}}}=z^{i}(\lambda)_{+}=\lambda^{3}+\frac{3}{2} u \lambda=\left(z^{2}-u\right)^{\frac{3}{2}}+\frac{3}{2} u \sqrt{z^{2}-u}
\end{gathered}
$$

to the formulas $(22)$. The generating differential (16) is now

$$
d S(z)=\sum t_{k} d \Omega_{k}(z)
$$

and the solution can be found in terms of the "periods"

$$
t_{k}=\frac{1}{k} \operatorname{res}_{\infty}\left(z^{-k} d S\right)
$$

For example the third $\mathrm{KdV}$ flow gives rise to

$$
\partial_{t_{3}} \Omega_{1}(z)=\partial_{t_{1}} \Omega_{3}(z)
$$

which is equivalent to the Hopf or dispersionless KdV equation

$$
u_{t_{3}}-\frac{3}{2} u u_{t_{1}}=0
$$

with generic solution

$$
t_{1}+\frac{3}{2} t_{3} u+P(u)=0 \quad P(u) \sim \sum t_{2 n+1} u^{n}
$$

Now, for the purposes of quantum field theory one needs to take from (29) the solution of the Whitham equation which is exact solution of the "full" KdV

$$
u=-\frac{2}{3} \frac{t_{1}}{t_{3}}
$$

The generating differential $(16)$ is now

$$
\left.d S\right|_{t_{3}=\frac{2}{3}, t_{k \neq 3}=0}=\lambda d\left(z^{2}(\lambda)\right)=-\left(\lambda^{2}+u\right) d \lambda+d(\ldots)
$$

Then the solution to the linear problem

$$
\left(\partial_{t_{1}}^{2}-\frac{2}{3} \frac{t_{1}}{t_{3}}\right) \Psi=z^{2} \Psi
$$

gives

$$
\begin{gathered}
\left.\Psi\left(t_{1}, z\right)\right|_{t_{3}=\frac{2}{3}}=A i\left(t_{1}+z^{2}\right) \\
\phi_{i}(z) \sim \frac{\partial^{i-1} \Psi(z, t)}{\partial t_{1}^{i-1}}=\sqrt{2 z} e^{-\frac{2}{3} z^{3}} \int d x x^{i-1} e^{-\frac{x^{3}}{3}+x z^{2}}
\end{gathered}
$$

and determinant formula [1]

$$
\frac{\mathcal{T}(t+T)}{\mathcal{T}(t)}=\frac{\operatorname{det} \phi_{i}\left(z_{j}\right)}{\Delta(z)}
$$

results in the $\tau$-function of the whole hierarchy in Miwa co-ordinates $T_{k}=-\frac{1}{k} \sum z_{j}^{-k}$. The decomposition of $\log \mathcal{T}(T)$ gives the correlators (2) of two-dimensional topological gravity. 


\section{Elliptic curves and the Gurevich-Pitaevsky solutions}

Now let us demonstrate that the similiar consideration of the global solutions related with higher genus Riemann surfaces (even in the elliptic case) gives rise to physically more interesting solutions. In contrast to the previous example Whitham times will be nontrivially related to the moduli of the curve. For example, from a standard definition (see [7]) in case of the elliptic curve $y^{2}=\prod_{i=1}^{3}\left(x-u_{i}\right)$ one has

$$
t_{k}=\frac{2}{k(2-k)} \operatorname{res}\left(x^{1-\frac{k}{2}} d y\right)
$$

or substituting $x=\wp(\xi)+c$ and $y=\frac{1}{2} \wp^{\prime}(\xi)$, where $\wp(\xi)$ is the Weierstrass $\wp$-function,

$$
\begin{gathered}
t_{k}=-\frac{2}{k(2-k)} \operatorname{res}\left((\wp(\xi)+c)^{1-\frac{k}{2}} \wp^{\prime \prime}(\xi) d \xi\right)= \\
=-\frac{6}{k(2-k)} \operatorname{Res} \frac{d \xi}{\xi^{6-k}}\left(1+c \xi^{2}+\frac{g_{2}}{20} \xi^{4}+\ldots\right)^{1-\frac{k}{2}}\left(1+\frac{g_{2}}{60} \xi^{4}+\ldots\right)= \\
=\frac{2}{5} \delta_{k, 5}-c \delta_{k, 3}+\left(\frac{3}{4} c^{2}-\frac{1}{4} g_{2}\right) \delta_{k, 1}+\mathcal{O}\left(t_{-k}\right)
\end{gathered}
$$

with dependent negative times $t_{-k}=t_{-k}\left(t_{1}, t_{3}\right)$ [9].

The elliptic (one-gap) solution to the KdV is

$$
\begin{aligned}
& U\left(t_{1}, t_{3}, \ldots \mid u\right)=\frac{\partial^{2}}{\partial t_{1}^{2}} \log \mathcal{T}\left(t_{1}, t_{3}, \ldots \mid u\right)= \\
& =U_{0 \wp}\left(k_{1} t_{1}+k_{3} t_{3}+\ldots+\Phi_{0} \mid \omega, \omega^{\prime}\right)+\frac{u}{3}
\end{aligned}
$$

and

$$
\begin{gathered}
d p \equiv d \Omega_{1}(z)=\frac{z-\alpha(u)}{y(z)} d z \\
d Q \equiv d \Omega_{3}(z)=\frac{z^{2}-\frac{1}{2} u z-\beta(u)}{y(z)} d z .
\end{gathered}
$$

Normalization conditions (12) prescribe that

$$
\alpha(u)=\frac{\oint_{A} \frac{z d z}{y(z)}}{\oint_{A} \frac{d z}{y(z)}} \quad \beta(u)=\frac{\oint_{A} \frac{\left(z^{2}-\frac{1}{2} u z\right) d z}{y(z)}}{\oint_{A} \frac{d z}{y(z)}} .
$$

The simplest elliptic example is the first Gurevich-Pitaevsky (GP) solution [10] with the underlying spectral curve

$$
y^{2}=\left(z^{2}-1\right)(z-u)
$$

specified by a requirement that all branching points except for $z=u$ are fixed and do not obey Whitham deformation. The generating differential corresponding to (40) is given by [4]

$$
d S(z)=\left(t_{1}+t_{3}\left(z+\frac{1}{2} u\right)+\ldots\right) \frac{z-u}{y(z)} d z \underset{\left\{t_{k>1}=0\right\}}{=} t_{1} \frac{z-u}{y(z)} d z
$$

and it produces the simplest solution to (15) coming from the elliptic curve. From (41) one derives:

$$
\begin{gathered}
\frac{\partial d S(z)}{\partial t_{1}}=\left(z-u-\left(\frac{1}{2} t_{1}+u t_{3}\right) \frac{\partial u}{\partial t_{1}}\right) \frac{d z}{y(z)} \\
\frac{\partial d S(z)}{\partial t_{3}}=\left(z^{2}-\frac{1}{2} u z-\frac{1}{2} u^{2}-\left(\frac{1}{2} t_{1}+u t_{3}\right) \frac{\partial u}{\partial t_{3}}\right) \frac{d z}{y(z)},
\end{gathered}
$$


and comparison with explicit expressions (38) implies:

$$
\begin{gathered}
\left(\frac{1}{2} t_{1}+u t_{3}\right) \frac{\partial u}{\partial t_{1}}=\alpha(u)-u, \\
\left(\frac{1}{2} t_{1}+u t_{3}\right) \frac{\partial u}{\partial t_{3}}=\beta(u)-\frac{1}{2} u^{2} .
\end{gathered}
$$

In other words, this construction provides the first GP solution to the Whitham equation

$$
\frac{\partial u}{\partial t_{3}}=v_{31}(u) \frac{\partial u}{\partial t_{1}}
$$

with

$$
v_{31}(u)=\frac{\beta(u)-\frac{1}{2} u^{2}}{\alpha(u)-u}=\left.\frac{d \Omega_{3}(z)}{d \Omega_{1}(z)}\right|_{z=u},
$$

which can be expressed through elliptic integrals 10 .

The elliptic solution with all moving branch points is given instead of (41) by

$$
y^{2}=\prod_{i=1}^{3}\left(z-u_{i}\right) \quad d S=y(z) d z
$$

and all $u_{i}$ are some functions of the Whitham times. Then one has to fulfil

$$
\begin{gathered}
d p=\frac{\partial}{\partial t_{1}} d S=y d z \sum_{i=1}^{3} \frac{v_{i}}{z-u_{i}}=\frac{z-\alpha(u)}{y} d z \\
d Q=\frac{\partial}{\partial t_{3}} d S=y d z \sum_{i=1}^{3} \frac{w_{i}}{z-u_{i}}=\frac{z^{2}-\left(\frac{1}{2} \sum u_{i}\right) z-\beta(u)}{y} d z
\end{gathered}
$$

where

$$
\begin{aligned}
v_{i} & =-\frac{1}{2} \frac{\partial u_{i}}{\partial t_{1}} \\
w_{i} & =-\frac{1}{2} \frac{\partial u_{i}}{\partial t_{3}}
\end{aligned}
$$

and $\alpha(u)$ and $\beta(u)$ are defined as before. Now what one gets is a simple linear system of the equations

$$
\begin{gathered}
R_{i j}(u) v_{j}=V_{i} \\
R_{i j}(u)=\left(\begin{array}{ccc}
1 & 1 & 1 \\
u_{2}+u_{3} & u_{1}+u_{3} & u_{1}+u_{2} \\
u_{2} u_{3} & u_{1} u_{3} & u_{1} u_{2}
\end{array}\right) \\
V^{T}=(0,-1,-\alpha(u)) \quad W^{T}=\left(1, \frac{1}{2}\left(u_{1}+u_{2}+u_{3}\right),-\beta(u)\right)
\end{gathered}
$$

The solution of linear system looks similiar to (44), for example:

$$
\frac{w_{1}}{v_{1}}=\frac{u_{1}^{2}-\frac{1}{2}\left(u_{1}+u_{2}+u_{3}\right)-\beta(u)}{u_{1}-\alpha(u)}
$$

and this class of solutions contain, for example, pure (non-topological!) $2 D$ gravity. The second GP solution 3

$$
y^{2}=\prod_{i=1}^{3}\left(z-u_{i}\right) \quad d S=(z-e) y d z
$$

${ }^{3}$ corresponding to the Yang-Lee edge singularity or $(2,5)$ conformal minimal model interacting with $2 D$ gravity 
can be obtained from elliptic curve with a marked point. In all these cases, one might expect that a "modulated" $\tau$-function (cf. with (11)) would still have a form

$$
\mathcal{T}\left\{t_{i}\right\}=e^{\mathcal{F}(t)} \vartheta(\mathbf{S}(t))
$$

so that

$$
\mathbf{k}_{i}(t)=\frac{\partial \mathbf{S}}{\partial t_{i}}
$$

and the poles of the "effective" potential

$$
u(t)=\frac{\partial^{2}}{\partial t_{1}^{2}} \log \mathcal{T}
$$

can be identified with the massive excitations (5).

\section{Toda-type curves}

The above procedure can be also applied to the finite-gap solutions determined by more general types of algebraic curves. The special case of the Toda-chain curves 19]

$$
y^{2}=P_{n}(z)^{2}-1 \quad 2 P_{n}(z)=w+w^{-1} \quad 2 y=w-w^{-1}
$$

was notices 4,12 , 13 to correspond to the $N=2 \mathrm{SYM}$ exact solutions with the gauge group $S U\left(n=N_{c}\right)$ t. The generating functional for the Toda-type theories is given by

$$
\begin{gathered}
d S=d z \log \left(P_{n}(z)+\sqrt{P_{n}(z)^{2}-1}\right) \\
d \Omega_{j}(z)=\frac{\partial d S}{\partial t_{j}}=\frac{d z}{\sqrt{P_{n}(z)^{2}-1}} \frac{\partial P_{n}(z)}{\partial t_{j}}
\end{gathered}
$$

and again corresponds to a specific Whitham solution. Indeed, for the $S L(2)$ case the generating differential (41) had a simple zero only in one of the branch points $z=u$, which means that the only branch point is influenced by the Whitham evolution. Now, it is easy to see that the zeroes of generating differential are at the points

$$
P_{n}(z)=1
$$

i.e. the Whitham dynamics is nontrivial only for the half of the branch points ( $y=0$ in (55)). In the special case of $n=1$ after the change of variables discussed in the footnote above the generating differential (56) acquires the form of 41 .

Now let us turn to the question what are the most general possible type of an integrable system appearing in the formulation of the exact quantum field theory solution. The pure Yang-Mills theory corresponds to the Toda-chain $(\mathrm{KdV})$ systems being the simplest ones among integrable models of this type. Their existing generalizations can be possible looked for in two different directions. One and the most obvious is towards the

\footnotetext{
${ }^{4}$ In fact one can show that the elliptic curve $[40$ is a particular case in the class of curves discussed below. Indeed if one takes

$$
w+w^{-1}=2 P_{1}(z)=2 z
$$
}

and considers the double cover

$$
z=u+x^{2}
$$

one gets (40) after the change of variables $y_{n e w}=y x[14,18$. 
Calogero-Moser particle systems and this is the case discussed from various points of view in [14, 17, 18, 16]. This line ends up at the free $N=4$ supersymmetric Yang-Mills theory which is described in terms of holomorphic generating differential $d S$ 局.

More interesting question is coupling of the $N=2$ SUSY YM theory with real matter, i.e. belonging to the fundamental representation of the gauge group. Such theories are supposed to be described by the following curves [2, 20]

$$
y^{2}=P_{n}(z)^{2}-P_{m}(z)
$$

where $m=N_{f}$ is the number of flavours or matter multiplets. These curves correspond to rather specific integrable systems such as Goryachev-Chaplygin top for $n=3$ and massless $m=2$. An integrable system corresponding to a generic curve can be constructed by standard methods of finite-gap integration 8 .

A generic integrable system of this type is determined by a (genus $g$ ) spectral curve, a meromorphic generating differential $d S$ and a set of $g$ given points $\left\{P_{1}, \ldots, P_{g}\right\}$ or initial conditions. The symplectic structure is defined by a (closed) form

$$
\Omega=\left.\sum_{\alpha=1}^{g} \frac{\partial}{\partial u_{\alpha}}\left(\frac{d S}{d z}\right)\right|_{z_{\alpha}=z\left(P_{\alpha}\right)} \delta u_{\alpha} \wedge \delta z_{\alpha}
$$

where as usual the moduli $\left\{u_{\alpha}\right\}$ for the hyperelliptic cases (46), (51), (55) and (58) can be taken as co-ordinates of the branch points. Any two functions of moduli $\left\{u_{\alpha}\right\}$ commute with respect to the Poisson bracket induced by (59) thus giving the set of $g$ independent Hamiltonians. The integrals

$$
\theta_{\alpha}=\sum_{\beta=1}^{g} \int_{z_{0}}^{z_{\alpha}} \frac{\partial d S}{\partial u_{\beta}}
$$

are the co-ordinates on the Jacobian of a spectral curve if $\frac{\partial d S}{\partial u_{\beta}}=d \omega_{\beta}$ are the holomorphic differentials.

The spectral curves (58) can be identically described by the equation

$$
\operatorname{det}(T(z)-w)=w^{2}-\operatorname{Tr} T(z) w+\operatorname{det} T(z)=0 \quad 2 y=w-w^{-1}
$$

where $T(z)$ is a monodromy matrix. The integrable system can be restored by constructing the Baker-Akhiezer function on a curve with marked points at the singularities of the monodromy matrix and the integrability can be proved using for example $r$-matrix formalism. In cases of (55), (58) the singular points are $P_{ \pm}=(\infty, \pm)$. The modulation of the integrals of motion again gives rise to the Whitham equations generated by

$$
d S=d z\left(\log \left(P_{n}(z)+\sqrt{P_{n}(z)^{2}-P_{m}(z)}\right)-\frac{1}{2} \log P_{m}(z)\right)
$$

\section{String equation. $\mathrm{KdV}$ case}

Finally, I will make few remarks concerning the way how in all the cases above a particular solution to the Whitham equations was distinguished. The idea is that "physical" solution to the Whitham hierarchy obeys

\footnotetext{
${ }^{5}$ The fact that the $N=4 \mathrm{SYM}$ theories are described in terms of holomorphic differentials can be easily understood assuming the connection of the Whitham hierarchy with the renormalization group approach. Indeed, on one hand the $N=4$ theory has zero $\beta$-functions and on the other hand it correspond to a trivial Whitham modulation. Since the holomorphic differentials have no singularities on spectral curve there is no natural way to introduce higher times of the $\mathrm{KP} /$ Toda type which is again consistent with the conformal invariance or trivial interaction with the "scale part" of metrics in $N=4$ theory.
} 
also some "string equation" which has a clear interpretation in terms of the spectral function for the auxiliary linear problem.

Let us consider the Lax equation for KdV

$$
\left(\partial_{x}^{2}+U(x)\right) \Psi(E, x)=E \Psi(E, x)
$$

as a Schrödinger equation. The partition function

$$
Z(t)=\operatorname{Tr} e^{-t \hat{H}}=\operatorname{Tr} e^{-t\left(\partial_{x}^{2}+U\right)}=\int_{x(0)=x(t)} D x e^{\int_{0}^{t} \dot{x}^{2}+U(x)}
$$

under the Laplace transform gives

$$
\rho(E)=\int_{0}^{\infty} d t e^{-t E} Z(t)=\operatorname{Tr} \frac{1}{\partial_{x}^{2}+U(x)-E}=\int d x \sum_{n \geq 0} \frac{R_{n}[U(x)]}{E^{n+\frac{1}{2}}}
$$

Now, using

$$
R_{n}[U(x)]=\frac{\partial^{2}}{\partial x \partial t_{2 n-1}} \log \mathcal{T}\left(x=t_{1}, t_{3}, \ldots\right)
$$

one can rewrite (66) as

$$
\begin{gathered}
\rho(E)=\sum_{n \geq 0} \frac{1}{E^{n+\frac{1}{2}}} \int d x \frac{\partial^{2}}{\partial x \partial t_{2 n-1}} \log \mathcal{T} \sim \\
\sim \sum_{n \geq 0} \frac{1}{E^{n+\frac{1}{2}}} \frac{\partial}{\partial t_{2 n-1}} \log \mathcal{T}\left(t_{k}-\frac{1}{k E^{\frac{k}{2}}}\right)
\end{gathered}
$$

or

$$
\rho(E)=\frac{\partial}{\partial E} \log \mathcal{T}\left(t_{k}-\frac{1}{k E^{\frac{k}{2}}}\right) \sim z^{q} \quad z=\sqrt{E}
$$

where the behaiviour $z^{q}$ is provided by string equation (specific initial condition), i.e. the solutions to string equations are characterized by growing at infinity spectral function and the order of the singularity at infinity gives a particular critical point.

The sense of the Whitham deformation is variation of the spectrum of the auxiliary linear problem (63). In terms of the path integral for the "spectral" fermions is

$$
\int D \psi^{*} D \psi e^{\int \psi^{*}\left(\partial_{x}^{2}+u(x)-E\right) \psi}=\operatorname{det}\left(\partial_{x}^{2}+u(x)-E\right)
$$

using the relation

$$
\log \operatorname{det}\left(\partial_{x}^{2}+U(x)-E\right)=\operatorname{Tr} \log \left(\partial_{x}^{2}+U(x)-E\right)
$$

and taking into account 65 ) one finds

$$
\frac{\partial}{\partial E} \log \operatorname{det}\left(\partial_{x}^{2}+U(x)-E\right)=\operatorname{Tr} \frac{1}{\partial_{x}^{2}+U(x)-E}=\rho(E)
$$

After integration the fermionic effective action

$$
\log \operatorname{det}\left(\partial_{x}^{2}+U(x)-E\right)=\int^{E} d \epsilon \rho(\epsilon)=S(E)
$$

coincides with the "Whitham effective action" $S(E)=\int^{E} d S$ in the sense of $\mathbb{7}, 21$. The total energy can be divided into fermionic and potential pieces

$$
E_{t o t}=\sum_{\text {fermions }} E+E_{\text {pot }}[U(x)]
$$


with measure of evaluating the sum in $(73)$ determined by a quasimomentum so that

$$
E_{f e r m}=\int E d p
$$

Now, the local extremum of (73) corresponds to the choice of a certain critical point if one takes

$$
E_{p o t}[U(x)]=\sum_{k \leq q} t_{k} \int d x R_{k}[U(x)]
$$

which gives a relation of the (35), (36) type. Thus we see that the quantum dynamics of the fermions entering the auxiliary linear problem gives rise to the deformation of spectral curve and nontrivial dynamics on its moduli space.

\section{Conclusion}

In this note I have tried to demonstrate the role played by the integrable equations in the nonperturbative approach to quantum string and supersymmetric gauge field theories. Using the general phenomenon that all known exact nonperturbative solutions can be described in terms of spectral curves and corresponding solutions to integrable systems one might hope that arising integrable equations of hydrodynamical type can be interpreted in terms of generalization of renormalization group beyond the perturbation theory. Amazingly enough this construction does not depend too much upon the "bare" quantum field theory, thus the language of integrable systems seems to be adequate and universal for studying nonperturbative phenomena.

\section{Acknowledgments}

I am deeply indebted to V.Fock, A.Gerasimov, A.Gurevich, S.Kharchev, A.Mironov, N.Nekrasov, S.Novikov, A.Zabrodin and especially to A.Gorsky, I.Krichever and A.Morozov for illuminating discussions. I am grateful to J.Ambjorn and A.Niemi for warm hospitality at Niels Bohr Institute and Institute of Theoretical Physics at Uppsala University where this work has been started and to the organizers of the conferences where these results were presented. The work was partially supported by the RFFI grant 95-01-01106, INTAS grant 93-0633 and by NFR-grant No F-GF 06821-305 of the Swedish Natural Science Research Council.

\section{References}

[1] R.Dijkgraaf, Preprint IASSNS-HEP-91/91

A.Marshakov, Int.J.Mod.Phys. A8 (1993) 3831

A.Morozov, Usp. Phys. Nauk 37 (1994) 1

P.Di Francesco, P.Ginsparg and J.Zinn-Justin, Phys.Rep. 254 (1995) 1

A.Mironov, Int.J.Mod.Phys. A9 (1994) 4355

B.Dubrovin, Geometry of 2D topological field theories, Preprint SISSA-89/94/FM

[2] N.Seiberg and E.Witten, Nucl.Phys. B426 (1994) 19

N.Seiberg and E.Witten, hepth/9408099 
A.Klemm, W.Lerche, S.Theisen and S.Yankelovich, hepth/9411048; hepth/9412158

P.Argyres and A.Farragi, hepth/9411057

[3] C.Vafa and E.Witten, hepth/9408074

[4] A.Gorsky, I.Krichever, A.Marshakov, A.Mironov and A.Morozov, hepth/9505035, Phys.Lett. B355 (1995) 466

[5] N.Hitchin, Duke.Math.Jour. 54 (1987) 91

[6] M.Kontsevich, Func.Anal.\& Apps., 25 (1991) 50

[7] I.Krichever, Func.Anal.\& Apps., 22 (1988),37

I.Krichever, Comm.Math.Phys. 143 (1991) 415; preprint LPTENS-92-18

[8] B.Dubrovin, I.Krichever and S.Novikov, Integrable systems - I, Sovremennye problemy matematiki (VINITI), Dynamical systems - 4 (1985) 179

[9] S.Kharchev, A.Marshakov, A.Mironov and A.Morozov., Mod.Phys.Lett. A8 (1993) 1047

[10] A.Gurevich and L.Pitaevsky, JETP, 65 (1973)65;

see also S.Novikov, S.Manakov, L.Pitaevsky and V.Zakharov "Theory of solitons"

[11] S.Cecotti and C.Vafa, Phys.Rev.Lett, 68 (1992) 903; Comm.Math.Phys. 158 (1993) 569

[12] E.Martinec and N.Warner, hepth/9509161

[13] T.Nakatsu and K.Takasaki, hepth/9509162

[14] R.Donagi and E.Witten, hepth/9510101

[15] T.Eguchi and C.Yang, hepth/9510183

[16] E.Martinec, hepth/9510204

[17] A.Gorsky and A.Marshakov, hepth/9510224

[18] H.Itoyama and A.Morozov, ITEP-M5/95, OU-HET-227, hepth/9511126

H.Itoyama and A.Morozov, ITEP-M6/95, OU-HET-230, hepth/9512161

[19] I.Krichever, in the Appendix to B.Dubrovin, Uspekhi Mat.Nauk, 36 (1981) N2, 12

[20] A.Hanany and Y.Oz, hepth/9505075

[21] S.Kharchev and A.Marshakov, Int.J.Mod.Phys. A10 (1995) 1219 